\title{
Association between -799C/T single nucleotide polymorphism of the MMP-8 promoter region and thoracic aortic dissection
}

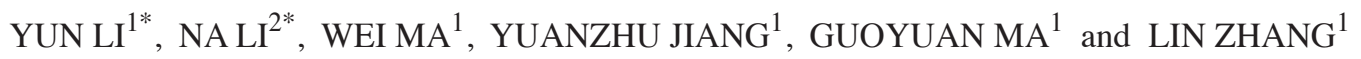 \\ ${ }^{1}$ Department of Thoracic Surgery, Shandong Provincial Hospital Affiliated to Shandong University, Jinan, Shandong 250021; \\ ${ }^{2}$ Department of Urology, Shandong Zhangqiu People's Hospital, Jinan, Shandong 250200, P.R. China
}

Received October 22, 2013; Accepted April 3, 2014

DOI: $10.3892 / \mathrm{mmr} .2014 .2437$

\begin{abstract}
Thoracic aortic dissection (TAD) is a life-threatening vascular condition, in which matrix metalloproteinases (MMPs) are involved. Since the key determinants underlying MMP action remain elusive, the present study investigated the correlation between single nucleotide polymorphisms (SNPs) in the promoter region of the MMP-8 gene and a predisposition to TAD, by comparing genotypes of TAD patients and healthy controls. From 154 TAD patients and 148 healthy individuals, DNA samples were obtained from venous blood, and genotyping was performed by a combination of polymerase chain reaction and automatic sequencing to detect SNPs in the MMP-8 promoter. Data were analyzed and odds ratios (OR) and $95 \%$ confidence intervals (CI) were calculated. $\mathrm{P}<0.05$ was considered to indicate a statistically significant result. Two SNPs, $-799 \mathrm{C} / \mathrm{T}$ and $-767 \mathrm{~A} / \mathrm{T}$, were identified in the MMP-8 promoter. Distribution of the -767A/T genotype was not significantly different between the patients and healthy controls. The $-799 \mathrm{C} / \mathrm{C}$ genotype was utilized as a match control, and significant differences in the genotypic distribution were observed between the patients with TAD and the controls. Furthermore, it was identified that the distribution of the $-799 \mathrm{C} / \mathrm{T}+\mathrm{T} / \mathrm{T}$ and $-799 \mathrm{C} / \mathrm{C}$ genotypes between the TAD and control populations was significantly different. The frequency of $\mathrm{T}$ allele distribution was higher in the TAD group (27\%) than in the control group (13.5\%). The genotype distribution followed the Hardy-Weinberg equilibrium. In the present study, it was concluded that the $-799 \mathrm{C} / \mathrm{T}$ polymorphism in the promoter region of MMP-8 may be associated with the development of TAD and that the T allele may increase patient predisposition to the disease.
\end{abstract}

Correspondence to: Professor Lin Zhang, Department of Thoracic Surgery, Shandong Provincial Hospital Affiliated to Shandong University, 324 Jing Wu Wei Qi Road, Jinan, Shangdong 250021, P.R. China

E-mail: zhanglindoctor@126.com

*Contributed equally

Key words: matrix metalloproteinase-8, single nucleotide polymorphism, thoracic aortic dissection

\section{Introduction}

Thoracic aortic dissection (TAD) is a life-threatening vascular condition, associated with a number of genetic factors that have been hypothesized to contribute to disease susceptibility (1). Recent studies on the genetic background of familial thoracic aortic aneurysms (TAAs) have focused on their association with connective tissue disorders, such as Marfan syndrome (2) and Ehlers-Danlos syndrome (3). Several studies have demonstrated that the development of aneurysmal disease may be linked to a number of genetic characteristics $(4,5)$. Up to $19 \%$ of patients with non-syndromic TAA have first-degree relatives affected by the disease, suggesting that genetic background is critical in the development of TAAs (6). Notably, environmental factors, including age, smoking, hypertension, hypercholesterolemia, and inflammatory and autoimmune disorders also significantly affect aortic structure, and thus also contribute to the development and progression of aortic disease (7).

The onset and development of TAD have been attributed to the breakdown of collagen and elastic fibers, loss of smooth muscle cells and accumulation of basophilic amorphous deposits (8). Although the histological characteristics of the dilated thoracic aorta may vary, they are underlined by the same pathophysiological mechanisms (9). Currently, studies investigating the processes involved in thoracic aorta aneurysms focus on the degradation of elastin, loss of smooth muscle cells and elevated concentrations of matrix metalloproteinases (MMPs) (10). Overexpression of MMPs produced by smooth muscle cells may contribute to the pathological alterations of the extracellular matrix (ECM) and therefore result in the dissection of the aorta (11). There is a prominent increase of MMP-8 and MMP-9 in the plasma of patients in the acute and subacute phases of TAD (12), as well as at the site of the ruptured aortic wall in patients with abdominal aneurysms (13). Furthermore, it has been demonstrated that the $-1562 \mathrm{C} / \mathrm{T}$ polymorphism in the promoter region of MMP-9 is associated with the development of aortic dissection with hyperpiesia, and that this polymorphism may increase patient predisposition to the condition (14).

A promoter is a specific DNA region that lies upstream of the gene and binds to transcription factors, and is therefore critically involved in the regulation of the gene and its subsequent protein expression. It was hypothesized that polymorphisms within the promoter region of MMP-8 may have an impact on the MMP- 8 transcription in patients with TAD. The majority 
Table I. Demographics and clinical characteristics of the TAD patients and controls.

\begin{tabular}{lcc}
\hline & TAD $(\mathrm{n}=154)$ & Normal controls $(\mathrm{n}=148)$ \\
\hline Age (mean \pm SD; years) & $54.6 \pm 11.4$ & $53.6 \pm 15.1$ \\
Male/female, $\mathrm{n}(\%)$ & $126 / 28(81.8 / 18.2)$ & $123 / 31(83.1 / 16.9)$ \\
$\mathrm{BMI}\left(\mathrm{kg} / \mathrm{m}^{2}\right)$ & $23.208 \pm 2.801$ & $24.876 \pm 3.198$ \\
Type A, $\mathrm{n}$ & 51 & - \\
Type B, $\mathrm{n}$ & 103 & - \\
Smokers, $\mathrm{n}(\%)$ & $53(34.4)$ & $40(27.0)$ \\
Hypertention, $\mathrm{n}(\%)$ & $130(84.6)$ & $47(31.8)$ \\
Diabetes mellitus, $\mathrm{n}(\%)$ & $15(9.7)$ & $21(14.1)$ \\
\hline
\end{tabular}

TAD, thoracic aortic dissection; BMI, body mass index; SD, standard deviation.

Table II. Primer sequences used for the amplification of the MMP-8 promoter.

\begin{tabular}{lccc}
\hline Primer name & \multicolumn{1}{c}{ Primer sequence } & Product size (bp) & Annealing temperature $\left({ }^{\circ} \mathrm{C}\right)$ \\
\hline Senseprimer1 & 5'-GCATCTCCCTCCCAAGGCTATGT-3' & 636 & 55 \\
Antisenseprimer1 & 5'-CCCTCCTGGCTTTCTTTCTGTCC-3' & & \\
Senseprimer2 & 5'-GAGACTACCATGCAGAGCCTATA-3' & 753 & 60 \\
Antisenseprimer2 & 5'-AAGGGACTAATCCTGAAGTTATGTG-3' & & \\
\hline
\end{tabular}

MMP-8, matrix metalloproteinase-8.

of previous studies on MMP polymorphisms have focused on MMP-8 polymorphisms involved in pulmonary disease (15), or the association between MMP-2, MMP-3, MMP-9 and MMP-12, and thoracic aortic aneurysms and TAD $(7,16,17)$. However, limited data are available regarding the association of MMP-8 polymorphisms with TAD.

In the present study, the correlation between single nucleotide polymorphisms (SNPs) in the MMP-8 promoter and TAD were investigated. For this, the promoter region of MMP-8 was amplified, sequenced and analyzed in a population comprised of patients with TAD and healthy individuals as the controls.

\section{Subjects and methods}

Patients. In total, 154 patients were selected with acute TAD that had been admitted to Shandong Provincial Hospital (Shandong, China) for participation in the present study. For all patients, the diagnosis was confirmed by enhanced computed tomography. The type of aortic dissection was determined according to the Stanford classification (18): 51 patients had type A and 103 patients had type B. Furthermore, 148 healthy individuals were selected as the reference control group from outpatients who complained of chest pain or underwent medical examinations, but did not have cancer, cardiovascular conditions or genetic diseases. All subjects were of Han ethnicity, with no familial relationship between them. Clinical variables included age, gender, type of dissection, body mass index (BMI), hyperpiesia, smoking habits and the presence of diabetes mellitus. Characteristics of patients with TAD and control individuals are listed in Table I. The study was approved by the Ethics
Committee of Shandong Provincial Hospital (Jinan, China) and all participants provided written informed consent.

Genotyping of SNPs in the MMP-8promoter. Blood samples were collected in EDTA-containing tubes and DNA was extracted using the Bloodready ${ }^{\mathrm{TM}}$ Multiplex PCR system (GenScript USA Inc., Piscataway, NJ, USA) according to the manufacturer's instructions. The MMP- 8 promoter fragments were amplified using two pairs of primers designed on human MMP-8 promoter sequences (GenBank accession no. AF059679). The primer sequences used are shown in Table II.

Polymerase chain reaction (PCR) was performed in a $20-\mu 1$ reaction volume containing $1 \mu \mathrm{l}$ genomic DNA, $4 \mu \mathrm{M}$ of each primer, $7.2 \mu \mathrm{l}$ water, $0.4 \mu \mathrm{l}$ dNTPs, $10 \mathrm{X}$ reaction buffer and $0.4 \mu 1$ Taq polymerase ('HotStart'Script ${ }^{\mathrm{TM}}$; GenScript USA Inc., Piscataway, NJ USA), and the insufficient part was filled up with water in the 9600 GeneAmp PCR thermal cycler (GenScript USA Inc.). The amplified fragments were sequenced using an ABI 377 sequencer (Applied Biosystems, Foster City, CA, USA) according to the manufacturer's instructions. The primer sequences, annealing temperatures and the sizes of the PCR products are presented in Table II. The reaction mixture was denatured for $1.5 \mathrm{~min}$ at $94^{\circ} \mathrm{C}$, annealed for $30 \mathrm{sec}$ at $55^{\circ} \mathrm{C}$ or at $60^{\circ} \mathrm{C}$, extended for $1 \mathrm{~min}$ at $72^{\circ} \mathrm{C}$ ( 35 cycles), and extended for another $5 \mathrm{~min}$ at $72^{\circ} \mathrm{C}$.

Statistical analysis. Data were analyzed using the SPSS version 13.0. statistical package for Windows (SPSS Inc. Chicago, IL, USA). Hardy-Weinberg equilibrium among control subjects was evaluated using observed genotype frequencies and 
Table III. Distribution of polymorphic site -799C/T in TAD patients and controls.

\begin{tabular}{|c|c|c|c|c|}
\hline & TAD patients $(n=154)$ & Controls $(n=148)$ & P-value & OR (95\%CI) \\
\hline \multicolumn{5}{|l|}{ Genotype } \\
\hline $\mathrm{C} / \mathrm{C}$ & $84(54.5 \%)$ & $113(76.4 \%)$ & & \\
\hline $\mathrm{C} / \mathrm{T}$ & $58(37.7 \%)$ & $30(20.3 \%)$ & 0.000 & $2.601(1.541-4.390)$ \\
\hline $\mathrm{T} / \mathrm{T}$ & $12(7.8 \%)$ & $5(3.3 \%)$ & 0.040 & $3.329(1.096-9.515)$ \\
\hline $\mathrm{C} / \mathrm{T}+\mathrm{T} / \mathrm{T}$ & $70(45.5 \%)$ & $35(23.6 \%)$ & 0.000 & $2.690(1.641-4.441)$ \\
\hline \multicolumn{5}{|l|}{ Allele } \\
\hline $\mathrm{C}$ & $226(73 \%)$ & $256(86.5 \%)$ & & \\
\hline $\mathrm{T}$ & $82(27 \%)$ & $40(13.5 \%)$ & 0.000 & $2.322(1.529-3.528)$ \\
\hline
\end{tabular}

Genotype (CC) as the reference group. TAD, thoracic aortic dissection; OR, odds ratio; CI, confidence interval.

ATG CAGAGCT TATAGTAGCTC C C

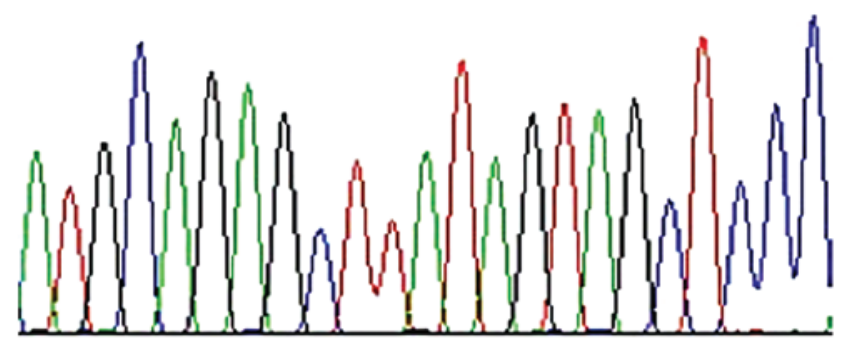

Figure 1. Peak pattern of -799C/T homozygote.

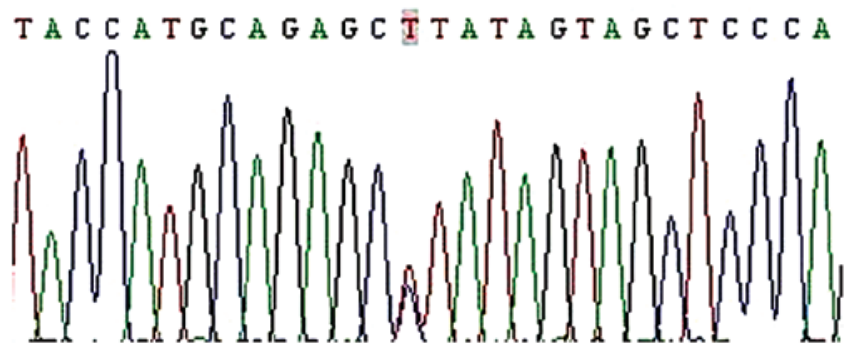

Figure 2. Peak pattern of $-799 \mathrm{C} / \mathrm{T}$ heterozygote.

the $\chi^{2}$ test. Differences between the patients and control groups were examined by the $\chi^{2}$ test by calculating the odds ratios (OR) and $95 \%$ confidence intervals $(95 \% \mathrm{CI}) . \mathrm{P}<0.05$ was considered to indicate a statistically significant difference.

\section{Results}

Allele and genotype distribution of $-799 \mathrm{C} / \mathrm{T}$ in patients and controls. Two SNPs, -799C/T and -767A/T, were identified in the MMP-8 promoter region (Figs. 1-4). The distribution of the $-799 \mathrm{C} / \mathrm{C},-799 \mathrm{C} / \mathrm{T}$ and $-799 \mathrm{~T} / \mathrm{T}$ genotypes was $54.5,37.7$ and $7.8 \%$ in the TAD group, and 76.4, 20.3 and 3.3\% in the control group, respectively. The $-799 \mathrm{C} / \mathrm{C}$ genotype was utilized as a reference group, and there was a significant difference in the genotype distribution between the TAD and control groups $(\mathrm{P}=0.000$ and 0.004, with an OR [95\% CI] of 2.601 [1.541-4.390] and 3.329

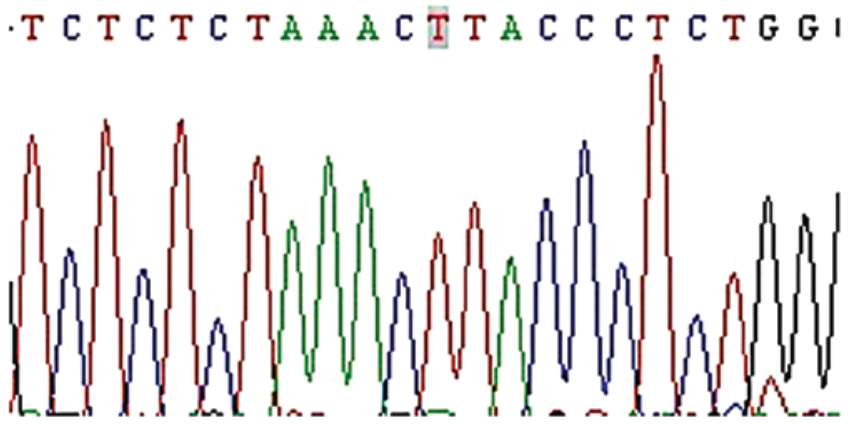

Figure 3. Peak pattern of -767A/T homozygote.

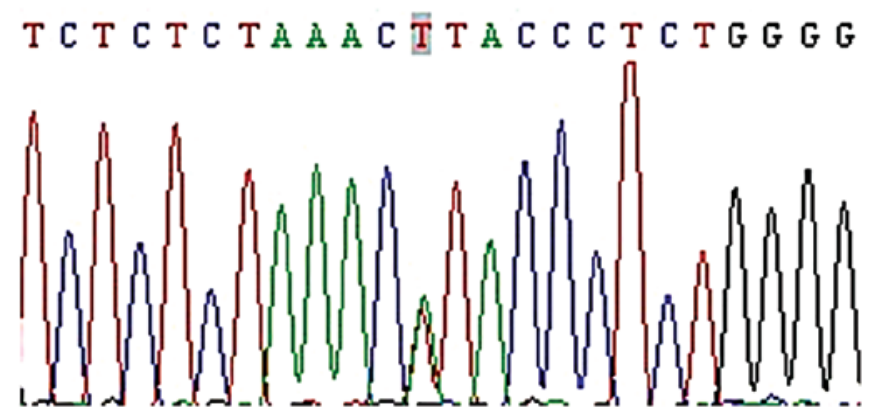

Figure 4. Peak pattern of -767A/T heterozygote.

[1.096-9.515], respectively). Furthermore, the distribution of $-799 \mathrm{C} / \mathrm{T}+\mathrm{T} / \mathrm{T}$ between the TAD and control individuals was also significantly different $(\mathrm{P}=0.000$ and $\mathrm{OR}$ [95\% CI] of 2.690 [1.641-4.441]). The $\mathrm{T}$ allele distribution in the TAD group was more frequent than that of the control group (27 vs. $13.5 \%$, with $\mathrm{P}=0.000$, and OR [95\% CI] of 2.322 [1.529-3.528]; Table III). The genotype distribution followed the Hardy-Weinberg equilibrium.

Allele and genotype distribution of $-767 \mathrm{~A} / \mathrm{T}$ in patients and controls. The distribution of the -767A/A, -767A/T and -767T/T genotypes was $81.8,16.9$ and $1.3 \%$ in the TAD population, and $84.5,13.5$ and $2.0 \%$ in the control population, respectively. There was no significant difference between the distribution of the $-767 \mathrm{~A} / \mathrm{T}$ and $-767 \mathrm{~T} / \mathrm{T}$ genotype in the TAD and control groups 
Table IV. The distribution of polymorphic site $-767 \mathrm{~A} / \mathrm{T}$ in TAD patients and controls.

\begin{tabular}{|c|c|c|c|c|}
\hline & TAD patients $(n=154)$ & Controls $(n=148)$ & P-value & OR $(95 \% \mathrm{CI})$ \\
\hline \multicolumn{5}{|l|}{ Genotype } \\
\hline $\mathrm{A} / \mathrm{A}$ & $126(81.8 \%)$ & $125(84.5 \%)$ & & \\
\hline $\mathrm{A} / \mathrm{T}$ & $26(16.9 \%)$ & $20(13.5 \%)$ & 0.430 & $1.290(0.685-2.429)$ \\
\hline $\mathrm{T} / \mathrm{T}$ & $2(1.3 \%)$ & $3(2.0 \%)$ & 1.000 & $0.661(0.109-4.026)$ \\
\hline $\mathrm{A} / \mathrm{T}+\mathrm{T} / \mathrm{T}$ & $28(18.2 \%)$ & $23(15.5 \%)$ & 0.540 & $1.208(0.660-2.211)$ \\
\hline \multicolumn{5}{|l|}{ Allele } \\
\hline A & $278(90.3 \%)$ & $270(91.2 \%)$ & & \\
\hline $\mathrm{T}$ & $30(9.7 \%)$ & $26(8.8 \%)$ & 0.685 & $1.121(0.646-1.945)$ \\
\hline
\end{tabular}

Genotype (AA) as the reference group. TAD, thoracic aortic dissection; OR, odds ratio; CI, confidence interval.

Table V. The distribution of polymorphic site $-799 \mathrm{C} / \mathrm{T}$ in patients with Stanford A and Stanford B.

\begin{tabular}{|c|c|c|c|c|c|}
\hline \multirow[b]{2}{*}{ Type } & \multirow[b]{2}{*}{$\mathrm{n}$} & \multicolumn{4}{|c|}{ Allele/genotype (\%) } \\
\hline & & $\mathrm{C}$ & $\mathrm{T}$ & $\mathrm{CC}$ & $\mathrm{CC}+\mathrm{CT}$ \\
\hline Stanford A & 51 & $78(76.5)$ & $24(23.5)$ & $31(60.7)$ & $20(39.3)$ \\
\hline Stanford B & 103 & $148(75.5)$ & $48(24.5)$ & $53(51.5)$ & $50(48.5)$ \\
\hline P-value & & \multicolumn{2}{|c|}{0.854} & \multicolumn{2}{|c|}{0.274} \\
\hline OR & & \multicolumn{2}{|c|}{0.949} & \multicolumn{2}{|c|}{0.684} \\
\hline $95 \% \mathrm{CI}$ & & \multicolumn{2}{|c|}{$0.541-1.663$} & \multicolumn{2}{|c|}{$0.346-1.353$} \\
\hline
\end{tabular}

Distribution of different allele/genotype (C and T; CC and CC+CT) between Stanford A and Stanford B patients compared with the P-values, OR and CI. OR, odds ratio; CI, confidence interval.

$(\mathrm{P}=0.430$ and 1.000; OR [95\% CI] of 1.290 [0.685-2.429] and 0.661 [0.109-4.026], respectively). Furthermore, the distribution of $-767 \mathrm{~A} / \mathrm{T}+\mathrm{T} / \mathrm{T}$ between the TAD and control groups was not significantly different $(\mathrm{P}=0.540$ and $\mathrm{OR}[95 \% \mathrm{CI}]$ of 1.208 [0.660-2.211]). Similarly, the distribution of the T allele was not significantly different between the TAD and control groups (9.7 vs. 8.8\%; $\mathrm{P}=0.685 ; \mathrm{OR}$ [95\% CI] of 1.121 [0.646-1.945]; Table IV). The genotypic distribution followed the Hardy-Weinberg equilibrium. Further analysis of the $-799 \mathrm{C} / \mathrm{T}$ polymorphic site by Stanford classification also revealed no significant differences (Table V).

\section{Discussion}

MMPs are members of a family of endopeptidases that process and degrade ECM proteins in connective tissues. MMPs are widely considered to be key regulatory factors in normal cellular physiology, as they are able to establish specialized microenvironments for cell differentiation, migration and invasion, and facilitate in the determination of cell survival and destruction (19). MMP-1, MMP-8 and MMP-13 are collagenases that degrade interstitial collagens of the ECM. The turnover and remodeling of the ECM is a highly regulated process, as uncontrolled proteolysis results in abnormalities that lead to the development of pathological diseases including TAA, which is characterized by excessive degradation of the ECM components.
To accomplish their normal or pathological function, MMPs must be produced by the appropriate cell type and within the correct pericellular region, in a time- and concentration-dependent manner. In addition, the activity of MMPs is highly regulated at the transcriptional, post-transcriptional (via mRNA stabilization), translational and post-translational (activation of the pro-MMP precursor zymogens) levels. Furthermore, MMP activity is regulated through the control of levels present within the tissue (by means of secretory regulation) or through the interaction with local tissue inhibitors (20).

Li et al (21) revealed a significant elevation in MMP-8 levels in the plasma of patients in the acute phase of TAD. This overexpression suggests that the polymorphisms altering MMP-8 expression contribute to patient susceptibility to TAD, however, the mechanisms underlying this effect remain unclear. Current studies are now focusing on genetic regulatory mechanisms, particularly the effect of SNPs on patient predisposition to TAD. For example, MMP gene expression may be affected by tumor necrosis factor- $\alpha$ (TNF- $\alpha$ ), through the activation of transcription factors that bind to specific response elements on the promoters of the MMP genes (22). Furthermore, genetic variations in the MMP- 8 promoter region may contribute to the MMP-8 expression, and thus, to increased risk of preterm premature rupture of fetal membranes (23).

Polymorphisms are DNA sequence variants that are present in at least $1 \%$ of the population when the variant is first observed. 
SNPs are the most common sequence variations in the human genome and occur in $\sim 1$ of every 1000 bases. SNPs occur in coding and non-coding regions of DNA, producing a variety of functional consequences. Due to the degenerate nature of the genetic code, not all SNPs have deleterious effects; however, SNPs in coding regions may alter the amino acid composition of proteins and reduce DNA binding, catalytic activity and receptor-ligand contact. SNPs in non-coding regions affect RNA processing, stability and translation, and SNPs in the 5'-untranscribed region may also affect transcription (24).

A study has revealed the presence of consensus sequences of nuclear binding proteins on the promoter regions of collagenase and stromelysin genes, including the phorbol ester-responsive element and AP-1 binding site (25). A promoter is a DNA region recognized by RNA polymerase that initiates gene transcription. In the process of RNA synthesis, the promoter interacts with transcription factors that initiate gene transcription and thus regulates protein expression. MMP-8 expression is mainly regulated at the transcriptional level, thus, SNPs in the promoter region promote gene transcription activity, resulting in significantly increased protein expression. It has been confirmed that expression of MMP-9 mRNA in patients with the $-1562 \mathrm{~T} / \mathrm{C}$ allele is five times higher than in those with the $\mathrm{C} / \mathrm{C}$ allele (26). These data demonstrated that mutations in the $\mathrm{T}$ allele promote the expression of MMPs.

In the present study, two SNPs, -799C/T and -767A/T, were identified in the MMP-8 promoter region. The frequencies of the $-799 \mathrm{C} / \mathrm{T}$ and $-799 \mathrm{~T} / \mathrm{T}$ genotypes in patients with TAD were significantly higher and the $\mathrm{T}$ allele distribution was also considerably elevated, as compared with that in the control subjects. Based on the calculated OR and $95 \%$ CI, it may be concluded that these SNPs correlate with the incidence of TAD and that patients with the T allele have a relatively higher predisposition to TAD. However, no correlation was identified between the -767A/T genotype and TAD.

In conclusion, these data demonstrated that while no correlation was observed between the -767A/T SNP and TAD, there was an evident association between the $-799 \mathrm{C} / \mathrm{T}$ polymorphism in the MMP- 8 gene and the incidence of TAD. Furthermore, the presence of the $\mathrm{T}$ allele may enhance susceptibility to aortic dissection. Understanding the genetic characteristics of TAD, will facilitate the improvement of diagnostic and treatment strategies for the future.

\section{Acknowledgements}

This study was supported by the Promotive Research Fund for Excellent Young and Middle-aged Scientisits of Shandong Province (grant no. BS2013YY066).

\section{References}

1. Jones GT, Phillips VL, Harris EL, Rossaak JI and van Rij AM: Functional matrix metalloproteinase-9 polymorphism (C-1562T) associated with abdominal aortic aneurysm. J Vasc Surg 38: 1363-1367, 2003.

2. Mizuguchi T, Collod-Beroud G, Akiyama T, Abifadel M, Harada N, Morisaki T, Allard D, Varret M, Claustres M, Morisaki H, et al: Heterozygous TGFBR2 mutations in Marfan syndrome. Nat Genet 36: 855-860, 2004.
3. Schwarze U,Schievink WI,Petty E,JaffMR,Babovic-Vuksanovic D, Cherry KJ, Pepin M and Byers PH: Haploinsufficiency for one COL3A1 allele of type III procollagen results in a phenotype similar to the vascular form of Ehlers-Danlos syndrome, Ehlers-Danlos syndrome type IV. Am J Hum Genet 69: 989-1001, 2001.

4. Deguara J, Burnand KG, Berg J, et al: An increased frequency of the 5A allele in the promoter region of the MMP3 gene is associated with abdominal aortic aneurysms. Hum Mol Genet 16: 3002-3007, 2007.

5. Jones GT, Phillips VL, Harris EL, Rossaak JI and van Rij AM: Functional matrix metalloproteinase-9 polymorphism (C-1562T) associated with abdominal aortic aneurysm. J Vasc Surg 38: 1363-1367, 2003.

6. Biddinger A, Rocklin M, Coselli J and Milewicz DM: Familial thoracic aortic dilatations and dissections: a case control study. J Vasc Surg 25: 506-511, 1997.

7. Chen L, Wang X, Carter SA, Shen YH, Bartsch HR, Thompson W, Coselli JS, Wilcken DL, Wang XL and LeMaire SA: A single nucleotide polymorphism in the matrix metalloproteinase 9 gene $(-8202 \mathrm{~A} / \mathrm{G})$ is associated with thoracic aortic aneurysms and thoracic aortic dissection. J Thorac Cardiovasc Surg 131: 1045-1052, 2006.

8. Barbour JR, Spinale FG and Ikonomidis JS: Proteinase systems and thoracic aortic aneurysm progression. J Surg Res 139: 292-307, 2007.

9. Hasham SN, Willing MC, Guo DC, Muilenburg A, He R, Tran VT, Scherer SE, Shete SS and Milewicz DM: Mapping a locus for familial thoracic aortic aneurysms and dissections (TAAD2) to 3p24-25. Circulation 107: 3184-3190, 2003.

10. Ikonomidis JS, Barbour JR, Amani Z, Stroud RE, Herron AR, McClister DM Jr, Camens SE, Lindsey ML, Mukherjee R and Spinale FG: Effects of deletion of the matrix metalloproteinase 9 gene on development of murine thoracic aortic aneurysms. Circulation 112 (Suppl): I242-248, 2005.

11. Ishii $\mathrm{T}$ and Asuwa $\mathrm{N}$ : Collagen and elastin degradation by matrix metalloproteinases and tissue inhibitors of matrix metalloproteinase in aortic dissection. Hum Pathol 31: 640-646, 2000.

12. Sangiorgi G, Trimarchi S, Mauriello A, Righini P, Bossone E, Suzuki T, Rampoldi V and Eagle KA: Plasma levels of metalloproteinases-9 and -2 in the acute and subacute phases of type A and type B aortic dissection. J Cardiovasc Med (Hagerstown) 7: 307-315, 2006.

13. Wilson WR, Anderton M, Schwalbe EC, Jones JL, Furness PN, Bell PR and Thompson MM: Matrix metalloproteinase- 8 and -9 are increased at the site of abdominal aortic aneurysm rupture. Circulation 113: 438-445, 2006.

14. Zhang B, Ye S, Herrmann SM, Eriksson P, de Maat M, Evans A, Arveiler D, Luc G, Cambien F, Hamsten A, et al: Functional polymorphism in the regulatory region of gelatinase $\mathrm{B}$ gene in relation to severity of coronary atherosclerosis. Circulation 99: 1788-1794, 1999.

15. González-Arriaga P, López-Cima MF, Fernández-Somoano A, Pascual T, Marrón MG, Puente XS and Tardón A: Polymorphism $+17 \mathrm{C} / \mathrm{G}$ in matrix metalloprotease MMP8 decreases lung cancer risk. BMC Cancer 8: 378, 2008.

16. Wagner S, Kluge B, Koziol JA, Grau AJ and Grond-Ginsbach C: MMP-9 polymorphisms are not associated with spontaneous cervical artery dissection. Stroke 35: e62-64, 2004.

17. Lamblin N, Bauters C, Hermant X, Lablanche JM, Helbecque N and Amouyel P: Polymorphisms in the promoter regions of MMP-2, MMP-3, MMP-9 and MMP-12 genes as determinants of aneurysmal coronary artery disease. J Am Coll Cardiol 40: 43-48, 2002.

18. Daily PO, Trueblood HW, Stinson EB, Wuerflein RD and Shumway NE: Management of acute aortic dissections. Ann Thorac Surg 10: 237-247, 1970

19. Allaire E, Hasenstab D, Kenagy RD, Starcher B, Clowes MM and Clowes AW: Prevention of aneurysm development and rupture by local overexpression of plasminogen activator inhibitor-1. Circulation 98: 249-255, 1998.

20. Barbour JR, Spinale FG and Ikonomidis JS: Proteinase systems and thoracic aortic aneurysm progression. J Surg Res 139: 292-307, 2007.

21. Li Y, Shao AZ, Jiang HT, Dong GH, Xu B, Yi J and Jing H: The prominent expression of plasma matrix metalloproteinase-8 in acute thoracic aortic dissection. J Surg Res 163: e99-104, 2010.

22. Noirey N, Staquet MJ, Gariazzo MJ, Serres M, André C, Schmitt D and Vincent C: Relationship between expression of matrix metalloproteinases and migration of epidermal and in vitro generated Langerhans cells. Eur J Cell Biol 81: 383-389, 2002. 
23. Wang H, Parry S, Macones G, Sammel MD, Ferrand PE, Kuivaniemi H, Tromp G, Halder I, Shriver MD, Romero R, et al: Functionally significant SNP MMP8 promoter haplotypes and preterm premature rupture of membranes (PPROM). Hum Mol Genet 13: 2659-2669, 2004.

24. Fleck TM, Koinig H, Czerny M, Hutschala D, Wolner E, Ehrlich M and Grabenwoger M: Impact of surgical era on outcomes of patients undergoing elective atherosclerotic ascending aortic aneurysm operations. Eur J Cardiothorac Surg 26: 342-347, 2004.
25. Birkedal-Hansen H, Moore WG, Bodden MK, Windsor LJ, Birkedal-Hansen B, DeCarlo A and Engler JA: Matrix metalloproteinases: a review. Crit Rev Oral Biol Med 4: 197-250, 1993.

26. Medley TL, Cole TJ, Dart AM, Gatzka CD and Kingwell BA: Matrix metalloproteinase-9 genotype influences large artery stiffness through effects on aortic gene and protein expression. Arterioscler Thromb Vasc Biol 24: 1479-1484, 2004. 